TRANSACTIONS OF THE

AMERICAN MATHEMATICAL SOCIETY

Volume 357, Number 4, Pages 1325-1339

S 0002-9947(04)03607-4

Article electronically published on April 27, 2004

\title{
PARAMETER-SHIFTED SHADOWING PROPERTY FOR GEOMETRIC LORENZ ATTRACTORS
}

\author{
SHIN KIRIKI AND TERUHIKO SOMA
}

\begin{abstract}
In this paper, we will show that any geometric Lorenz flow in a definite class satisfies the parameter-shifted shadowing property.
\end{abstract}

\section{INTRODUCTION}

We will study the problem whether there exists a definite class of geometric Lorenz flows which can be depicted as accurately as one desires. Theoretically, such an accurate depiction is guaranteed by the shadowing property. However, Komuro 9] showed that geometric Lorenz flows do not satisfy the (parameterfixed) shadowing property except in very restricted cases. So, we need to consider our problem under a somewhat relaxed condition, which is the parameter-shifted shadowing property in our case.

The geometric Lorenz model is one of important examples in dynamical systems, which was studied in the initial stages by Guckenheimer and Williams [6, 19, 7, 20], Afraimovich, Bykov and Shil'nikov [1] and Yorke and Yorke [21]. Their aim was to construct topologically a simple mechanism which can give results similar to that of the parametrized ODE system in $\mathbb{R}^{3}$ presented experimentally by Lorenz [10]. For some parameter values, Lorenz observed typical characters of chaotic motions in butterfly-shaped attractors. The question whether or not the original Lorenz system for such parameter values has the same structure as the geometric Lorenz model has been unsolved for more than 30 years. By combination of normal form theory and rigorous computations, Tucker [17] answered this question affirmatively, that is, for classical parameters, the original Lorenz system has a robust strange attractor which is given by the same rules as for the geometric Lorenz model. From these facts, we know that the geometric Lorenz model is crucial in the study of Lorenz dynamical systems. See Viana [18 for more information.

The first return map on a Poincaré cross section of a geometric Lorenz flow is a Lorenz map $L: \Sigma \backslash \Gamma \longrightarrow \Sigma$, where $\Sigma=\left\{(x, y) \in \mathbb{R}^{2} ;|x|,|y| \leq 1\right\}$ and $\Gamma=\left\{(0, y) \in \mathbb{R}^{2} ;|y| \leq 1\right\}$. So, we will first prove the parameter-shifted shadowing property (PSSP) for Lorenz maps.

Received by the editors April 10, 2003 and, in revised form, July 31, 2003

2000 Mathematics Subject Classification. Primary 37C50, 37D45, 37D50; Secondary 34C28.

Key words and phrases. Geometric Lorenz model, strange attractor, shadowing property.

The first author was supported in part by Research Institute for Science and Technology at TDU Grant Q02J-02, Q03J-08. 
Theorem A. There exists a definite set $\mathcal{L}$ of Lorenz maps satisfying the following condition:

- For any $L \in \mathcal{L}$ and any $\varepsilon>0$, there exist $\mu>0$ and $\delta>0$ such that any $\delta$-pseudo orbit of the Lorenz map $L_{\mu}$ with $L_{\mu}(x, y)=L(x, y)-(\mu x, 0)$ is $\varepsilon$-shadowed by an actual orbit of $L$.

The strict description of $\mathcal{L}$ is given in the next section.

In the case when any elements in a one-parameter family $\left\{f_{\mu}\right\}_{\mu \in I}$ are naturally defined maps, "PSSP for $f=f_{0}$ " means that any $\delta$-pseudo-orbit for $f$ is $\varepsilon$-shadowed by an actual orbit of $f_{\mu}$ for some $\mu \in I$. This idea was first introduced by Coven, Kan and Yorke [5] and Nusse and Yorke [15] in some one-dimensional dynamics. See also Kiriki and Soma [8] for PSSP for Lozi maps. In the present case, $L_{\mu}$ 's other than the original $L$ are artificially defined maps. We wish here to describe actual orbits of the given map $L$ as accurately as possible but not those of auxiliary maps $L_{\mu}, \mu>0$. Thus, we adopt as our definition of PSSP for $L$ that any $\delta$-pseudo-orbit for $L_{\mu}$ is $\varepsilon$-shadowed by an actual orbit of $L$.

As an application of Theorem A, we have the following result, which is our main theorem.

Theorem B. Any geometric Lorenz flow controlled by a Lorenz map $L \in \mathcal{L}$ has the parameter-shifted shadowing property.

See the next section for the definition of the parameter-shifted shadowing property of Lorenz flows.

\section{Preliminaries}

Let $\Sigma_{ \pm}$denote the components of $\Sigma \backslash \Gamma$ with $\Sigma_{ \pm} \ni( \pm 1,0)$. A map $L: \Sigma \backslash \Gamma \longrightarrow \Sigma$ is said to be a Lorenz map if it is a piecewise $C^{1}$ diffeomorphism which has the form

$$
L(x, y)=(\alpha(x), \beta(x, y)),
$$

where $\alpha:[-1,1] \backslash\{0\} \rightarrow[-1,1]$ is a piecewise $C^{1}$-map with symmetric property $\alpha(-x)=-\alpha(x)$ and satisfying

$$
\begin{cases}\lim _{x \rightarrow 0+} \alpha(x)=-1, & \alpha(1)<1, \\ \lim _{x \rightarrow 0+} \alpha^{\prime}(x)=\infty, & \alpha^{\prime}(x)>\sqrt{2} \text { for any } x \in(0,1]\end{cases}
$$

(see Figure 1(a)), and $\beta: \Sigma \backslash \Gamma \longrightarrow[-1,1]$ is a contraction in the $y$-direction. Moreover, it is required that the images $L\left(\Sigma_{+}\right), L\left(\Sigma_{-}\right)$are mutually disjoint cusps in $\Sigma$, where the vertices $\mathbf{v}_{+}, \mathbf{v}_{-}$of $L\left(\Sigma_{ \pm}\right)$are contained in $\{\mp 1\} \times[-1,1]$ respectively; see Figure 1(b).

Now, we introduce the notion of the shadowing property for Lorenz planar maps.

Definition 2.1. For $\delta>0$, a sequence $\left\{\mathbf{x}_{n}\right\}_{n \geq 0} \subset \Sigma$ is called a $\delta$-pseudo-orbit of a Lorenz map $L$ if

$$
\left|L\left(\mathbf{x}_{n}\right)-\mathbf{x}_{n+1}\right| \leq \delta
$$

for any integer $n \geq 0$. Here, we suppose that if $\mathbf{x}_{n} \in \Gamma$, then $\mathbf{x}_{n+1}$ is contained in one of the $\delta$-neighborhoods of $\mathbf{v}_{+}$and $\mathbf{v}_{-}$.

Definition 2.2. A Lorenz map $L$ has the parameter-shifted shadowing property, for short PSSP, if there exists a one-parameter family $\left\{L_{\mu}\right\}_{\mu \in I}$ of Lorenz maps with 


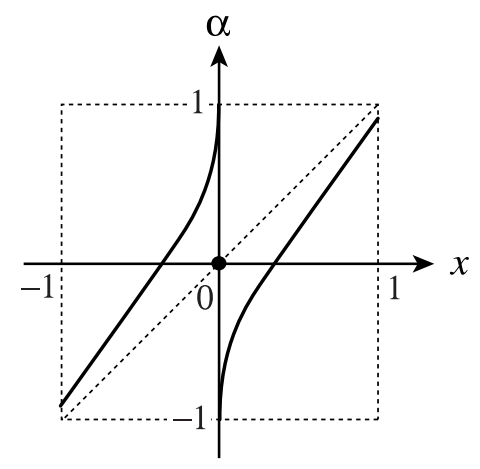

(a)

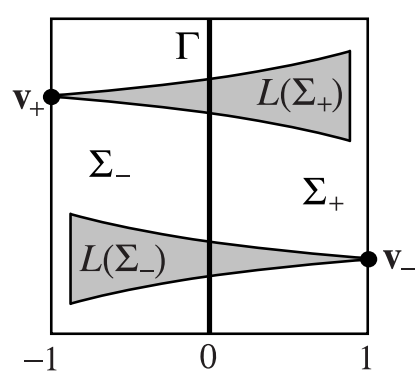

(b)

FiguRE 1.

$I=\left[0, \mu_{0}\right]$ for $0 \leq \mu_{0}<1$ satisfying the following conditions (i) and (ii):

(i) $L_{0}=L$.

(ii) For any $\varepsilon>0$, there exist $\delta>0$ and $\mu \in I$ such that any $\delta$-pseudo-orbit $\left\{\mathbf{x}_{n}\right\}_{n \geq 0}$ of $L_{\mu}$ is $\varepsilon$-shadowed by an actual orbit of $L$, i.e., there exists a $\mathbf{z} \in \Sigma \backslash \Gamma$ such that

$$
\left|L^{n}(\mathbf{z})-\mathbf{x}_{n}\right| \leq \varepsilon
$$

for any $n \geq 0$.

When the parameter of $\left\{L_{\mu}\right\}_{\mu \in I}$ is fixed, i.e., $I=\{0\}$, the definition of PSSP is identical to that of the original (parameter-fixed) shadowing property given in [2]. According to Komuro [9, Theorem 1], $L$ has the parameter-fixed shadowing property only when $\alpha(1)=1$; see also [16]. In our case, since $\alpha(1)<1$ by (2.1), any Lorenz map $L$ does not have the original shadowing property.

We are mainly concerned with Lorenz maps $L(x, y)=(\alpha(x), \beta(x, y))$ satisfying the following extra conditions (2.2)-(2.4):

$$
\begin{gathered}
\left|\frac{\partial \beta}{\partial x}(x, y)\right|,\left|\frac{\partial \beta}{\partial y}(x, y)\right|<\frac{3}{4 \sqrt{2}} \text { for any }(x, y) \in \Sigma \backslash \Gamma, \\
0.8<\alpha^{2}(1)<\alpha(1)<1, \\
\alpha^{\prime}(x)<2 \quad \text { for any } x \text { with } 0.8<x \leq 1 .
\end{gathered}
$$

These conditions are not so severe, and it is not hard for us to construct various Lorenz maps satisfying them practically. In the condition (2.2), we took the concrete value $3 /(4 \sqrt{2})$ in order to simplify the proof of the theorem below. In fact, one can prove the theorem under the weaker assumption

$$
\sup _{(x, y) \in \Sigma \backslash \Gamma}\left\{\left|\frac{\partial \beta}{\partial x}(x, y)\right|,\left|\frac{\partial \beta}{\partial y}(x, y)\right|\right\}<\frac{1}{\sqrt{2}} .
$$

The following is the precise statement of Theorem A.

Theorem 2.3 (PSSP for Lorenz planar maps). Any Lorenz map $L$ with the conditions (2.2)-2.4 admits a one-parameter family $\left\{L_{\mu}\right\}_{\mu \in I}$,

$$
L_{\mu}(x, y)=L(x, y)-(\mu x, 0),
$$




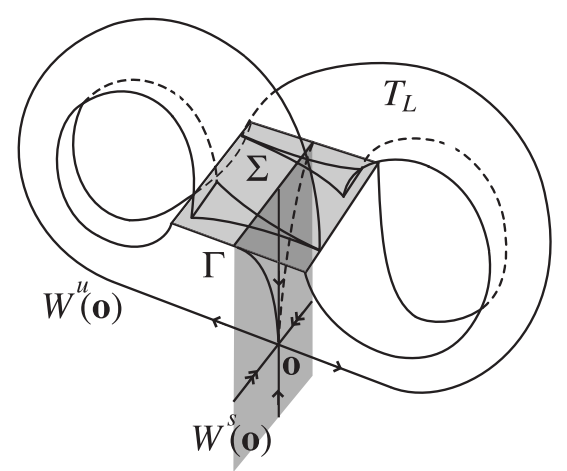

FiguRE 2.

satisfying the parameter-shifted shadowing property. Precisely, for any $\varepsilon>0$, there exist $\delta>0$ and $\mu \in I$ so that the following (i) and (ii) hold:

(i) Any infinite $\delta$-pseudo-orbit $\left\{\mathbf{x}_{n}\right\}_{n=1}^{\infty}$ of $L_{\mu}$ (possibly $\left.\mathbf{x}_{n} \in \Gamma\right)$ is $\varepsilon$-shadowed by the actual orbit $\left\{L^{n}(\mathbf{z})\right\}_{n=1}^{\infty}$ of $L$ for some $\mathbf{z} \in \Sigma$ with $\bigcup_{n=0}^{\infty}\left\{L^{n}(\mathbf{z})\right\} \cap \Gamma=$ $\emptyset$.

(ii) Any finite $\delta$-pseudo-orbit $\left\{\mathbf{x}_{n}\right\}_{n=1}^{m}$ of $L_{\mu}$ with $\mathbf{x}_{m} \in \Gamma$ is $\varepsilon$-shadowed by the actual orbit $\left\{L^{n}(\mathbf{z})\right\}_{n=1}^{m}$ of $L$ for some $\mathbf{z} \in \Sigma$ with $\bigcup_{n=0}^{m-1}\left\{L^{n}(\mathbf{z})\right\} \cap \Gamma=\emptyset$ and $L^{m}(\mathbf{z}) \in \Gamma$.

Let us identify $\Sigma$ with $\left\{(x, y, 1) \in \mathbb{R}^{3} ;|x|,|y| \leq 1\right\}$, and $\Gamma$ with $\{(0, y, 1) \in$ $\left.\mathbb{R}^{3} ;|y| \leq 1\right\}$. A $C^{1}$-vector field $X_{L}$ on $\mathbb{R}^{3}$ is said to be a geometric Lorenz vector field controlled by a Lorenz map $L: \Sigma \backslash \Gamma \longrightarrow \Sigma$ if it satisfies the following conditions (i) and (ii):

(i) For any point $(x, y, z)$ in a neighborhood of the origin $\mathbf{0}$ of $\mathbb{R}^{3}, X_{L}$ is given by $(\dot{x}, \dot{y}, \dot{z})=\left(\lambda_{1} x,-\lambda_{2} y,-\lambda_{3} z\right)$, where $\lambda_{i}$ are positive numbers satisfying $\lambda_{3}<\lambda_{1}<\lambda_{2}$. Moreover, $\Gamma$ is contained in the stable manifold $W^{s}(\mathbf{0})$ of $\mathbf{0}$.

(ii) All forward orbits of $X$ starting from $\Sigma \backslash \Gamma$ will return to $\Sigma$ and the first return map is $L$.

Note then that $\mathbf{0}$ is a singular point (an equilibrium) of saddle type, the local unstable manifold of $\mathbf{0}$ is tangent to the $x$-axis, and the local stable manifold of $\mathbf{0}$ is tangent to the $y z$-plane as shown in Figure 2, A $C^{1}$-map $\varphi_{L}: \mathbb{R}^{3} \times \mathbb{R} \rightarrow \mathbb{R}^{3}$ is the geometric Lorenz flow controlled by $L$ (for short $L$-Lorenz flow) if it generated by $X_{L}$, i.e., $\varphi_{L}(\mathbf{x}, 0)=\mathbf{x}$ and $(\partial / \partial t) \varphi_{L}(\mathbf{x}, t)=X_{L}\left(\varphi_{L}(\mathbf{x}, t)\right)$. The closure of $\bigcup_{\mathbf{z} \in \Sigma \backslash \Gamma} \varphi_{L}(\mathbf{z},[0, \infty))$ in $\mathbb{R}^{3}$ is homeomorphic to the genus two solid handlebody as illustrated in Figure 2] which is called a trapping region of $\varphi_{L}$ and denoted by $T_{\varphi_{L}}$ or $T_{L}$. Any forward orbit for $\varphi_{L}$ with an initial point in $T_{L}$ cannot escape from $T_{L}$. The invariant set $\bigcap_{t>0} \varphi_{L}\left(T_{L}, t\right)$ for $X_{L}$ does not have any continuous hyperbolic splitting at $\mathbf{0}$, but it belongs to an essential class called singular hyperbolic, which is studied extensively from various approaches by Morales, Pacifico and others; see for details [3, 4, 11, 12, 13, 14]. 
Now, we introduce the notion of PSSP for Lorenz flows.

Definition 2.4. Let $\psi$ be a geometric Lorenz flow, and $\delta, \tau$ positive numbers.

(i) A sequence $\left\{\mathbf{x}_{n}\right\}_{n \geq 0}$ in $T_{\psi}$ with $\mathbf{x}_{0} \in \Sigma$ is a $(\delta, \tau)$-pseudo-orbit for the flow $\psi$ if there exists a sequence $\left\{\tau_{n}\right\}_{n \geq 0}$ such that, for any $n \geq 0$,

$$
\tau \leq \tau_{n} \leq 2 \tau \text { and }\left|\psi\left(\mathbf{x}_{n}, \tau_{n}\right)-\mathbf{x}_{n+1}\right| \leq \delta .
$$

For each $n \geq 0$, we set

$$
\Psi_{n}=\psi\left(\mathbf{x}_{n},\left[0, \tau_{n}\right]\right)
$$

and call $\left\{\Psi_{n}\right\}_{n \geq 0}$ the $(\delta, \tau)$-chain for $\psi$ associated to $\left\{\mathbf{x}_{n}\right\}_{n \geq 0}$ (or more strictly to $\left.\left.\left\{\mathbf{x}_{n} ; \tau_{n}\right\}_{n>0}\right\}\right)$.

(ii) The $(\delta, \tau)$-chain $\left\{\Psi_{n}\right\}_{n>0}$ is said to be $\varepsilon$-shadowed by a flow $\varphi$ if there exists a point $\mathbf{y} \in \Sigma$ and a surjective $C^{1}$-diffeomorphism $h:[0, \infty) \longrightarrow[0, \infty)$ satisfying

$$
\left|\varphi(\mathbf{y}, h(t))-\psi\left(\mathbf{x}_{n}, t-\sum_{i=0}^{n-1} \tau_{i}\right)\right| \leq \varepsilon
$$

for any $t \geq 0$ with $\sum_{i=0}^{n-1} \tau_{i} \leq t \leq \sum_{i=0}^{n} \tau_{i}$. Then, we also say that $\left\{\Psi_{n}\right\}_{n \geq 0}$ is $\varepsilon$-shadowed by $\varphi$ with $\varphi(\mathbf{y}, t) ; t \geq 0$.

Remark 2.5. In Definition 2.4(i), the upper bound condition $\tau_{n} \leq 2 \tau$ is not essential, but added for our convenience. When $\tau_{n}>2 \tau$ for some $n$, split $\left[0, \tau_{n}\right]$ into subintervals $\left[\tau_{n}^{(i-1)}, \tau_{n}^{(i)}\right](i=1, \cdots, k)$ with $\tau_{n}^{(0)}=0, \tau_{n}^{(k)}=\tau_{n}$ and $\tau \leq$ $\tau_{n}^{(i)}-\tau_{n}^{(i-1)} \leq 2 \tau$. Then, the expanded sequence of $\left\{\mathbf{x}_{n}\right\}_{n \geq 0}$ obtained by adding the entries $\mathbf{x}_{n}^{(i)}=\psi\left(\mathbf{x}_{n}, \tau_{n}^{(i)}\right)(i=1, \cdots, k-1)$ between $\mathbf{x}_{n}$ and $\mathbf{x}_{n+1}$ defines a $(\delta, \tau)$-pseudo-orbit for $\psi$ in the sense of Definition 2.4(i); see Figure 3

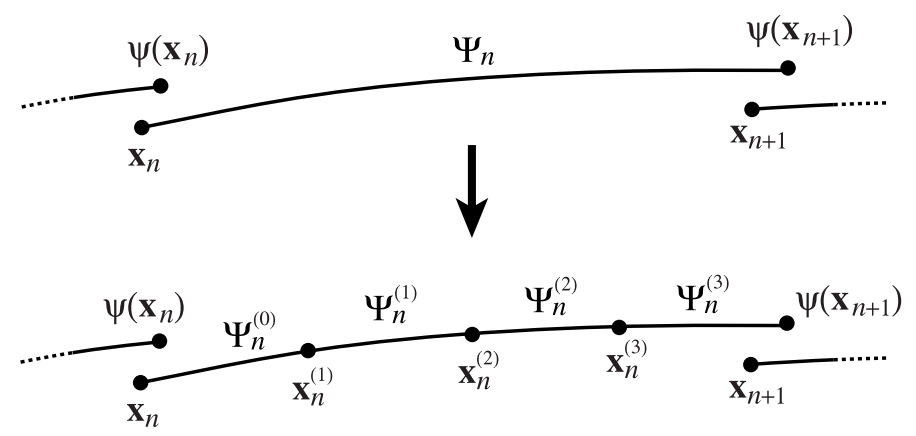

FIGURE 3.

Definition 2.6. We say that a given geometric Lorenz flow $\varphi$ (or the vector field generating $\varphi$ ) has the parameter-shifted shadowing property if there exists a $C^{1}$ one-parameter family $\left\{\varphi_{\mu}\right\}_{\mu \in\left[0, \mu_{0}\right]}$ of geometric Lorenz flows such that

(i) $\varphi_{0}=\varphi$;

(ii) for any $\varepsilon>0$, there exist $\delta, \tau>0$ and $\mu \in\left[0, \mu_{0}\right]$ such that any $(\delta, \tau)$-chain for $\varphi_{\mu}$ is $\varepsilon$-shadowed by $\varphi_{0}$.

The following is the precise statement of Theorem B. 
Theorem 2.7 (PSSP for Lorenz flows). Any geometric Lorenz flow controlled by a Lorenz map satisfying the conditions (2.2)-(2.4) has the parameter-shifted shadowing property.

Remark 2.8 (Absence of strong PSSP for Lorenz flows). Our PSSP for Lorenz flows is the weak one in the sense of Definition 3 in 9 . We say that a $(\delta, \tau)$-chain $\left\{\Phi_{\mu ; n}\right\}_{n \geq 0}$ for $\varphi_{L_{\mu}}$ is strongly $\varepsilon$-shadowed by $\varphi_{L}$ with $\varphi_{L}(\mathbf{y}, h(t))_{t \geq 0}$ if a diffeomorphism $h:[0, \infty) \longrightarrow[0, \infty)$ as in Definition 2.4 satisfies the extra condition: $\left|h^{\prime}(t)-1\right|<\varepsilon$ for any $t \geq 0$. However, any $\varphi_{L}$ as in Theorem 2.7 has a constant $\varepsilon=\varepsilon(L)>0$ such that, for any $\delta, \tau>0$ and any $\mu \in I$ (possibly $\mu=0$ ), there exists a $(\delta, \tau)$-chain for $\varphi_{L_{\mu}}$ which is not strongly $\varepsilon$-shadowed by any actual flow of $\varphi_{L}$. This implies that $\varphi_{L}$ does not have the strong PSSP. In fact, one can define a $(\delta, \tau)$-pseudo-orbit $\left\{\mathbf{x}_{n}\right\}_{n \geq 0}$ in $T_{L_{\mu}}$ for $\varphi_{L_{\mu}}$ such that, in a small neighborhood of $\mathbf{0}$ in $\mathbb{R}^{3}$, the sequence satisfies $\mathbf{x}_{n_{0}}=\mathbf{x}_{n_{0}+1}=\cdots=\mathbf{x}_{n_{0}+m}$ for an arbitrarily large $m \geq 0$. Such a sequence $\left\{\mathbf{x}_{n}\right\}_{n \geq 0}$ is not strongly $\varepsilon$-shadowed by $\varphi_{L}$. The proof is elementary but somewhat tedious, so we will omit it.

\section{PSSP FOR LORENZ PLANAR MAPS}

Let $L: \Sigma \backslash \Gamma \longrightarrow \Sigma$ with $L(x, y)=(\alpha(x), \beta(x, y))$ be a Lorenz map satisfying the conditions (2.1)-(2.4). For any $\mu>0$, consider the function $\alpha_{\mu}:[-1,1] \backslash\{0\} \longrightarrow \mathbb{R}$ defined by

$$
\alpha_{\mu}(x)=\alpha(x)-\mu x .
$$

If $\mu_{0}=\mu_{0}(\alpha)>0$ is sufficiently small, then for any $\mu \in\left[0, \mu_{0}\right], \alpha_{\mu}$ is a function with $\alpha_{\mu}([-1,1] \backslash\{0\}) \subset[-1,1]$ and satisfies (2.1), (2.3) and (2.4). Set $I=\left[0, \mu_{0}\right]$. Then, we have the one-parameter family $\left\{L_{\mu}\right\}_{\mu \in I}$ of Lorenz maps with

$$
L_{\mu}(x, y)=\left(\alpha_{\mu}(x), \beta(x, y)\right)
$$

for $(x, y) \in \Sigma \backslash \Gamma$.

By the condition (2.3), there exists $0<\eta_{0}<1$ such that, for any interval $J=[-\eta, 0]$ or $[0, \eta]$ with $0<\eta \leq \eta_{0}$ and any $\mu \in\left[0, \mu_{0}\right]$,

$$
\bigcup_{i=1}^{3} \alpha_{\mu}^{i}(J) \subset[0.8,1] \text {. }
$$

For any $\varepsilon>0$, we set

$$
\varepsilon_{1}=\min \left\{3 \mu_{0}, \frac{\eta_{0}}{8}, \frac{\varepsilon}{64}\right\} \quad \text { and } \delta=\frac{\varepsilon_{1}}{100} .
$$

Proposition 3.1. The map $\widehat{\alpha}=\alpha_{\varepsilon_{1} / 3}$ satisfies the following (i) and (ii):

(i) Any infinite $\delta$-pseudo-orbit $\left\{x_{n}\right\}_{n=0}^{\infty}$ of $\widehat{\alpha}$ is $\varepsilon / 8$-shadowed by an actual orbit $\left\{\alpha^{n}(z)\right\}_{n=0}^{\infty}$ of $\alpha$ for some $z \in[-1,1]$ with $\bigcup_{n=0}^{\infty}\left\{\alpha^{n}(z)\right\} \not \supset 0$.

(ii) Any finite $\delta$-pseudo-orbit $\left\{x_{n}\right\}_{n=0}^{m}$ of $\widehat{\alpha}$ with $x_{m}=0$ is $\varepsilon / 8$-shadowed by an actual orbit $\left\{\alpha^{n}(z)\right\}_{n=0}^{m}$ of $\alpha$ for some $z \in[-1,1]$ with $\bigcup_{n=0}^{m-1}\left\{\alpha^{n}(z)\right\} \not \supset 0$ and $\alpha^{m}(z)=0$.

Consider any infinite $\delta$-pseudo-orbit $\left\{x_{n}\right\}_{n=0}^{\infty}$ of $\widehat{\alpha}$. Let $l_{0}$ be the closed interval in $\mathbb{R}$ with $o\left(l_{0}\right)=x_{0}$ and $\left|l_{0}\right|=2 \varepsilon_{1}$, where $\left|l_{0}\right|$ is the length of $l_{0}$ and $o\left(l_{0}\right)$ is the center of $l_{0}$. For the proof of Proposition 3.1, we will define a certain sequence of closed intervals $\left\{l_{n}\right\}_{n \geq 0}$ in $[-1,1]$ with $\operatorname{Int} l_{n} \cap\{0\}=\emptyset$ and $\alpha\left(l_{n}\right) \supset l_{n+1}$, where the notation $\operatorname{Int} l_{n}$ means the interior of $l_{n}$. 
Suppose first that $l_{0} \cap\{0\}=\emptyset$. We may assume that $l_{0} \subset(0,1]$. Then, $\widehat{\alpha}\left(l_{0}\right)$ is the closed interval in $[-1,1]$ such that $\widehat{\alpha}\left(x_{0}\right)$ divides $\widehat{\alpha}\left(l_{0}\right)$ into two intervals of length at least $\sqrt{2} \varepsilon_{1}$. Since $0 \leq \alpha(x)-\widehat{\alpha}(x)=\left(\varepsilon_{1} / 3\right) x \leq \varepsilon_{1} / 3$ for any $x \in(0,1]$, $\alpha\left(l_{0}\right)$ is obtained from $\widehat{\alpha}\left(l_{0}\right)$ by $\left(0, \varepsilon_{1} / 3\right)$-RHS-shifting (see Figure 4 ), where we say that for two closed intervals $l=[e, f], l^{\prime}=\left[e^{\prime}, f^{\prime}\right]$ and $0 \leq \gamma \leq \eta, l^{\prime}$ is obtained from $l$ by $(\gamma, \eta)$-right-hand-side shifting (for short $(\gamma, \eta)$-RHS-shifting) if $\gamma \leq e^{\prime}-e \leq \eta$ and $\gamma \leq f^{\prime}-f \leq \eta$.

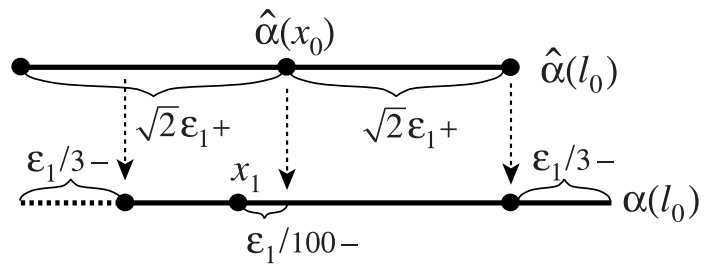

Figure 4. " $\sqrt{2} \varepsilon_{1}+$ " (resp. " $\varepsilon_{1} / 3-$ ") in the figure means that the length between the corresponding points is at least $\sqrt{2} \varepsilon_{1}$ (resp. at most $\left.\varepsilon_{1} / 3\right)$. These rules are applied in any figures below.

Since $\left|x_{1}-\widehat{\alpha}\left(x_{0}\right)\right| \leq \varepsilon_{1} / 100$, the distance between $x_{1}$ and either end point of $\alpha\left(l_{0}\right)$ is at least $\sqrt{2} \varepsilon_{1}-\varepsilon_{1} / 3-\varepsilon_{1} / 100>\varepsilon_{1}$. Thus, the interval $l_{1}$ with $\left|l_{1}\right|=2 \varepsilon_{1}$ and $o\left(l_{1}\right)=x_{1}$ is contained in the interior of $\alpha\left(l_{0}\right)$. If $l_{1} \cap\{0\}=\emptyset$, one can define $l_{2} \subset \operatorname{Int} \alpha\left(l_{1}\right)$ with $\left|l_{2}\right|=2 \varepsilon_{1}$ and $o\left(l_{2}\right)=x_{2}$ similarly.

Suppose next that $l_{0} \cap\{0\} \neq \emptyset$. We may assume that $x_{0} \leq 0$ and $\widehat{\alpha}\left(x_{0}\right)>0$. Set $l_{0}^{-}=l_{0} \cap[-1,0]$. Then, $\widehat{\alpha}\left(l_{0}^{-}\right)$is the closed interval in $[0.8,1]$ containing 1 ; see Figure 5 (a). Note that the distance between $\widehat{\alpha}\left(x_{0}\right)$ and the end point of $\widehat{\alpha}\left(l_{0}^{-}\right)$other than 1 is at least $\sqrt{2} \varepsilon_{1}$. The interval $\widehat{\alpha}\left(l_{0}^{-}\right)$is obtained from $\alpha\left(l_{0}^{-}\right)$by $\left(0, \varepsilon_{1} / 3\right)$-RHSshifting. Since $\left|\widehat{\alpha}\left(x_{0}\right)-x_{1}\right| \leq \varepsilon_{1} / 100$, the interval $l_{1}=\left[x_{1}-(\sqrt{2}-1 / 100) \varepsilon_{1}, x_{1}\right]$ is contained in $\alpha\left(l_{0}^{-}\right)$. Note that, from the condition (3.1), $\bigcup_{i=0}^{2}\left(\alpha^{i}\left(l_{1}\right) \cup \widehat{\alpha}^{i}\left(l_{1}\right)\right)$ is contained in $[0.8,1]$. Since $\alpha^{\prime}(x)>\sqrt{2}$ for any $x \in(0,1]$, the length of the interval $\alpha^{3}\left(l_{1}\right)$ is at least $2 \sqrt{2}(\sqrt{2}-1 / 100) \varepsilon_{1}>3.9 \varepsilon_{1}$. Since $0.8(2+\sqrt{2}+1) \varepsilon_{1} / 3>1.1 \varepsilon_{1}$ and $\left(2^{2}+2+1\right) \varepsilon_{1} / 3<2.4 \varepsilon_{1}, \alpha^{3}\left(l_{1}\right)$ is obtained from $\widehat{\alpha}^{3}\left(l_{1}\right)$ by $\left(1.1 \varepsilon_{1}, 2.4 \varepsilon_{1}\right)$-RHS shifting; see Figure 5(b). Since $\alpha^{\prime}(x)<2$ for any $x \in[0.8,1]$,

$$
\left|\widehat{\alpha}^{3}\left(x_{1}\right)-x_{4}\right| \leq\left(2^{2}+2+1\right) \frac{\varepsilon_{1}}{100}<0.1 \varepsilon_{1} .
$$

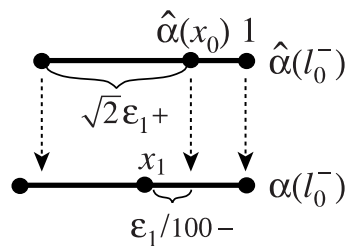

(a)

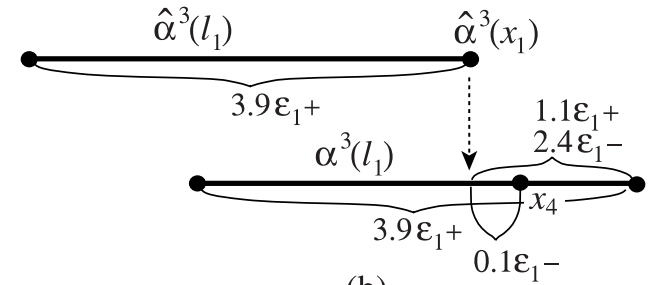

(b)

Figure 5. 
Thus, the closed interval $l_{4}$ with $\left|l_{4}\right|=2 \varepsilon_{1}$ and $o\left(l_{4}\right)=x_{4}$ is contained in $\operatorname{Int} \alpha^{3}\left(l_{1}\right)$. Set $l_{2}=\alpha\left(l_{1}\right)$ and $l_{3}=\alpha\left(l_{2}\right)$. Then, $\left|l_{2}\right|<\left|l_{3}\right| \leq 2^{2}\left|l_{1}\right|<6 \varepsilon_{1}$ and, for $i=1,2$,

$$
\begin{aligned}
\left|\alpha^{i}\left(x_{1}\right)-x_{i+1}\right| & \leq\left|\alpha^{i}\left(x_{1}\right)-\widehat{\alpha}^{i}\left(x_{1}\right)\right|+\left|\widehat{\alpha}^{i}\left(x_{1}\right)-x_{i+1}\right| \\
& \leq(2+1)\left(\frac{\varepsilon_{1}}{3}+\frac{\varepsilon_{1}}{100}\right)<1.1 \varepsilon_{1} .
\end{aligned}
$$

In particular, for any $y \in l_{i},\left|y-x_{i}\right| \leq 6 \varepsilon_{1}+1.1 \varepsilon_{1}<8 \varepsilon_{1}$. Now, for any given $\left\{x_{n}\right\}_{n=0}^{\infty}$ of $\widehat{\alpha}$, we get the sequence of closed intervals $\left\{l_{n}\right\}_{n \geq 0}$ with $\operatorname{Int} l_{n} \cap\{0\}=\emptyset$ and $\alpha\left(l_{n}\right) \supset l_{n+1}$. For any $n \geq 0$, we set $l_{n+1}^{(n)}=\alpha^{-1}\left(l_{n+1}\right) \cap l_{n}$. For any $m>n+1$, $l_{m}^{(n)}$ can be defined inductively on $m-n$ by $l_{m}^{(n)}=\alpha^{-1}\left(l_{m}^{(n+1)}\right) \cap l_{n}$. Note that the restriction $\alpha^{m-n} \mid l_{m}^{(n)}: l_{m}^{(n)} \longrightarrow l_{m}$ is a bijection.

Lemma 3.2 is proved by applying the argument above repeatedly.

Lemma 3.2. There exists a sequence $\left\{l_{n}\right\}_{n=0}^{\infty}$ of closed intervals satisfying the following conditions (i)-(iv):

(i) $\varepsilon_{1} \leq\left|l_{n}\right| \leq 6 \varepsilon_{1}$.

(ii) For any $y \in l_{n},\left|y-x_{n}\right| \leq 8 \varepsilon_{1}$.

(iii) If $l_{n}$ is not contained in $[-1,-0.8] \cup[0.8,1]$, then $\left|l_{n}\right|=2 \varepsilon_{1}$ and $o\left(l_{n}\right)=x_{n}$.

(iv) For any $n \geq 0, \alpha\left(l_{n}\right)$ contains $l_{n+1}$ and there exists $m>n$ with $l_{m}^{(n)} \subset \operatorname{Int} l_{n}$. Moreover, if $l_{n} \ni 0$, then $\operatorname{Int} l_{n+1}^{(n)} \cap\{0\}=\emptyset$.

The proof of Proposition 3.1 follows easily from Lemma 3.2

Proof of Proposition 3.1. (i) Since $l_{0} \supset l_{1}^{(0)} \supset l_{2}^{(0)} \supset \cdots$, the intersection $\bigcap_{n=1}^{\infty} l_{n}^{(0)}$ is non-empty. Take a point $z \in \bigcap_{n=1}^{\infty} l_{n}^{(0)}$. Since $\alpha^{n}(z) \in l_{n}$ for any $n \geq 0$, by Lemma 3.2

$$
\left|\alpha^{n}(z)-x_{n}\right| \leq 8 \varepsilon_{1} \leq \varepsilon / 8
$$

If $0 \in l_{n}$, then $\alpha^{n}(z) \in l_{m}^{(n)} \subset \operatorname{Intl}_{n+1}^{(n)} \subset l_{n}$ for some $m>n+1$. This implies $\alpha^{n}(z) \neq 0$ for any $n \geq 0$.

(ii) Assuming that $\left\{x_{n}\right\}_{n=1}^{m}$ is a finite subsequence of an infinite $\delta$-pseudo-orbit of $\widehat{\alpha}$, we have a sequence $l_{0}, l_{1}, \cdots, l_{m}$ of closed intervals satisfying the conditions (i)-(iv) of Lemma 3.2. If $x_{m}=0$, then $l_{m}$ is the interval with $\left|l_{m}\right|=2 \varepsilon_{1}$ and $o\left(l_{m}\right)=0$. In particular, $0 \in \operatorname{Int} l_{m}$. Thus, $\bigcap_{n=1}^{m} l_{m}^{(0)}$ contains a unique point $z$ with $\alpha^{n}(z) \neq 0$ for $0 \leq n \leq m-1$ and $\alpha^{m}(z)=0$. This completes the proof.

Proof of Theorem 2.3. (i) Let $\left\{\mathbf{x}_{n}\right\}_{n=1}^{\infty}$ be any $\delta$-pseudo-orbit for $L_{\mu}$ with $\mu=$ $\varepsilon_{1} / 3$. Set $\left[\mathbf{x}_{n}\right]_{x}=x_{n}$ and $\left[\mathbf{x}_{n}\right]_{y}=y_{n}$, where $[\mathbf{w}]_{x},[\mathbf{w}]_{y}$ denote respectively the $x$ and $y$-coordinates of a point $\mathbf{w} \in \Sigma$. Then, $\left\{x_{n}\right\}_{n=1}^{\infty}$ is a $\delta$-pseudo-orbit for $\widehat{\alpha}$. By Proposition 3.1(i), there exists $z \in[-1,1]$ such that $\left\{\alpha^{n}(z)\right\}_{n=1}^{\infty} \varepsilon / 8$-shadows $\left\{x_{n}\right\}_{n=1}^{\infty}$. If we set $\mathbf{z}=\left(z, y_{0}\right)$, then

$$
\left|\mathbf{z}-\mathbf{x}_{0}\right|=\left|z-x_{0}\right|<\varepsilon .
$$

Suppose that $\left|L^{n}(\mathbf{z})-\mathbf{x}_{n}\right|<\varepsilon$ for $n=0,1, \cdots, m$. Let $J$ be a straight segment in $\Sigma$ connecting $L^{m}(\mathbf{z})$ with $\mathbf{x}_{m}$. If $c:[0, \nu] \longrightarrow J$ is an arc-length parametrization of $J$, then $|\dot{c}|^{2}=\dot{c}_{1}^{2}+\dot{c}_{2}^{2}=1$ and $\nu<\varepsilon$, where $\dot{c}=(d / d t) c$ and $c(t)=\left(c_{1}(t), c_{2}(t)\right)$. Since $\left|\alpha^{m}(z)-x_{m}\right|<8 \varepsilon_{1} \leq \eta_{0}$, Int $J$ is disjoint from $\Gamma$. In fact, if Int $J \cap \Gamma$ were not empty, by (3.1), then $\left|\alpha^{m+1}(z)-\alpha\left(x_{m}\right)\right|>2(1-|[0.8,1]|)=1.6$. This contradicts 
the following fact:

$$
\begin{aligned}
\left|\alpha^{m+1}(z)-\alpha\left(x_{m}\right)\right| \leq & \left|\alpha^{m+1}(z)-x_{m+1}\right|+\left|x_{m+1}-\widehat{\alpha}\left(x_{m}\right)\right| \\
& +\left|\widehat{\alpha}\left(x_{m}\right)-\alpha\left(x_{m}\right)\right|<8 \varepsilon_{1}+\delta+\frac{\varepsilon_{1}}{3} .
\end{aligned}
$$

Thus, $L_{\mu} \circ c:[0, \nu] \longrightarrow \Sigma$ is a continuous path connecting $L_{\mu}\left(L^{m}(\mathbf{z})\right)$ with $L_{\mu}\left(\mathbf{x}_{m}\right)$. For any $t \in(0, \nu)$,

$$
\frac{d}{d t}\left(L_{\mu} \circ c\right)(t)=\left(\frac{\partial \widehat{\alpha}}{\partial x}(c(t)) \dot{c}_{1}(t), \frac{\partial \beta}{\partial x}(c(t)) \dot{c}_{1}(t)+\frac{\partial \beta}{\partial y}(c(t)) \dot{c}_{2}(t)\right) .
$$

By the condition (2.2),

$$
\left|\left[L_{\mu}\left(L^{m}(\mathbf{z})\right)\right]_{y}-\left[L_{\mu}\left(\mathbf{x}_{m}\right)\right]_{y}\right| \leq \frac{3}{4 \sqrt{2}} \cdot \sqrt{2} \nu<\frac{3 \varepsilon}{4},
$$

where the " $\sqrt{2}$ " of $\sqrt{2} \nu$ is derived from the fact that the maximum of $u+v$ is $\sqrt{2}$ under the assumption of $u^{2}+v^{2}=1$. Note that $\left[L_{\mu}\left(L^{m}(\mathbf{z})\right)\right]_{y}=\left[L^{m+1}(\mathbf{z})\right]_{y}$, and

$$
\left|\left[L_{\mu}\left(\mathbf{x}_{m}\right)\right]_{y}-y_{m+1}\right| \leq\left|L_{\mu}\left(\mathbf{x}_{m}\right)-\mathbf{x}_{m+1}\right|<\delta
$$

It follows that $\left|\left[L^{m+1}(\mathbf{z})\right]_{y}-y_{m+1}\right|<7 \varepsilon / 8$. Since $\left|\left[L^{m+1}(\mathbf{z})\right]_{x}-x_{m+1}\right|=\mid \alpha^{m+1}(z)-$ $x_{m+1}|<\varepsilon / 8,| L^{m+1}(\mathbf{z})-\mathbf{x}_{m+1} \mid<\varepsilon$. Thus, $\left\{\mathbf{x}_{n}\right\}_{n=1}^{\infty}$ is $\varepsilon$-shadowed by $\left\{L^{n}(\mathbf{z})\right\}_{n=1}^{\infty}$.

The proof of (ii) is done similarly by using Proposition 3.1(ii).

\section{PSSP FOR LORENZ FLOWS}

In this section, we will prove Theorem [2.7. First, consider a Lorenz map $L$ satisfying the conditions (2.2) $-(2.4)$ and an $L$-Lorenz flow $\varphi$. Recall that $\Gamma=$ $\{(0, y, 1) \in \Sigma ;|y| \leq 1\}$ is the singularity set on $\Sigma$, and set $\tilde{\Gamma}=\{(x, y, z) \in \Pi ; x=$ $0\}$, where $\Pi=[-1,1]^{2} \times[0,1]$. For the proof of PSSP for $\varphi$, we need to fix a oneparameter family of Lorenz maps $L_{\mu}$ and $L_{\mu}$-Lorenz flows. Here, we suppose that $\left\{L_{\mu}\right\}_{\mu \in\left[0, \mu_{0}\right]}$ is the one-parameter family given in $\S 2$ and take a $C^{1}$-one-parameter family $\left\{\varphi_{\mu}\right\}_{\mu \in\left[0, \mu_{0}\right]}$ satisfying $\partial \varphi_{\mu} / \partial t(\mathbf{x}, 0)=\partial \varphi / \partial t(\mathbf{x}, 0)$ for any $\mathbf{x} \in N_{1 / 10}(\tilde{\Gamma}, \Pi)$, where $N_{\eta}(Y, X)$ denotes the $\eta$-neighborhood of a compact subset $Y$ in a metric space $(X, d)$, that is, $N_{\eta}(Y, X)=\{x \in X ; d(x, Y) \leq \eta\}$.

Let us fix $0<\varepsilon<1$ arbitrarily and determine constants $\hat{\delta}, \hat{\tau}>0$ and $\hat{\mu} \in\left[0, \mu_{0}\right]$ such that any $(\hat{\delta}, \hat{\tau})$-chain for $\varphi_{\hat{\mu}}$ is $\varepsilon$-shadowed by $\varphi$.

4.1. Interpolated chains and crossing sequences. Throughout the remainder of this section, fix $\hat{\tau}>0$ so that, for any $\mathbf{x} \in \Sigma$ and $\mu \in\left[0, \mu_{0}\right], \varphi_{\mu}(\mathbf{x},(0,5 \hat{\tau}]) \cap \Sigma=\emptyset$. Let $\left\{\mathbf{x}_{n}\right\}_{n \geq 0}$ be a $(\delta, \hat{\tau})$-pseudo-orbit for $\varphi_{\mu}$, i.e., $\left|\mathbf{x}_{n+1}-\varphi_{\mu}\left(\mathbf{x}_{n}, t_{n}\right)\right| \leq \delta$ for some $\left\{t_{n}\right\}_{n \geq 0}$ with $\hat{\tau} \leq t_{n} \leq 2 \hat{\tau}$ and $\mathbf{x}_{0} \in \Sigma$. Let $\left\{\Phi_{\mu ; n}\right\}_{n \geq 0}$ be the $(\delta, \hat{\tau})$-chain for $\varphi_{\mu}$ associated to $\left\{\mathbf{x}_{n}\right\}_{n \geq 0}$, i.e., $\Phi_{\mu ; n}=\varphi_{\mu}\left(\mathbf{x}_{n},\left[0, t_{n}\right]\right)$. When $\varphi_{\mu}\left(\mathbf{x}_{n}, t_{n}\right) \neq \mathbf{x}_{n+1}, \sigma_{n}$ is the open segment in $\mathbb{R}^{3}$ whose closure connects $\varphi_{\mu}\left(\mathbf{x}_{n}, t_{n}\right)$ with $\mathbf{x}_{n+1}$, and otherwise $\sigma_{n}=\emptyset$. Set

$$
\tilde{\Phi}_{\mu ; n}=\Phi_{\mu ; n} \cup \sigma_{n},
$$

and call $\left\{\tilde{\Phi}_{\mu ; n}\right\}_{n \geq 0}$ the interpolated $(\delta, \hat{\tau})$-chain for $\varphi_{\mu}$ associated to $\left\{\mathbf{x}_{n}\right\}_{n \geq 0}$ (or more strictly to $\left\{\mathbf{x}_{n}, t_{n}\right\}_{n \geq 0}$ ). Let $U$ be a small neighborhood of the origin in $\mathbf{R}^{3}$ with $U \cap \Sigma=\emptyset$. When $\Phi_{\mu ; n}$ is contained in $U, \Phi_{\mu ; n}$ may have an arbitrarily small length. On the other hand, $\Phi_{\mu ; n}$ 's not contained in $U$ have lengths bounded away from zero. Thus, there exists $\delta_{0}>0$ such that, for any $\Phi_{\mu ; n}$ with $\Phi_{\mu ; n} \cap \Sigma \neq \emptyset$, the length of $\Phi_{\mu ; n}$ is greater than $3 \delta_{0}$. This assumption is crucial in our argument 
below. In fact, it guarantees that, if $0<\delta \leq \delta_{0}$, the union $\bigcup_{n>0} \tilde{\Phi}_{\mu ; n}$ of any interpolated $(\delta, \hat{\tau})$-chain contains no jagged subsets intersecting $\Sigma$ zigzag. So, we suppose from now on that $0<\delta \leq \delta_{0}$.

Let $\left\{n_{i}\right\}_{i \geq 0}$ be the strictly monotone increasing sequence with $n_{0}=0$ and such that $\left\{n_{i}\right\}_{i \geq 1}$ consists of all positive integers $n$ with $\tilde{\Phi}_{\mu ; n} \cap \Sigma \neq \emptyset$ and $\tilde{\Phi}_{\mu ; n-1} \cap \Sigma=\emptyset$.

Definition 4.1. For each $n_{i} \geq 0$, the crossing point $\mathbf{y}_{i}$ of $\tilde{\Phi}_{\mu ; n_{i}}$ is a unique point of $\Phi_{\mu ; n_{i}} \cap \Sigma$ if $\Phi_{\mu ; n_{i}} \cap \Sigma \neq \emptyset$ (see Figure [6(a)), otherwise $\mathbf{y}_{i}$ is a point of $\sigma_{n_{i}} \cap \Sigma$ (see Figure [6 b)). The $\left\{\mathbf{y}_{i}\right\}_{i \geq 0}$ is called the $(\delta, \hat{\tau})$-crossing sequence for $\varphi_{\mu}$ associated to $\left\{\mathbf{x}_{n}\right\}_{n \geq 0}$.

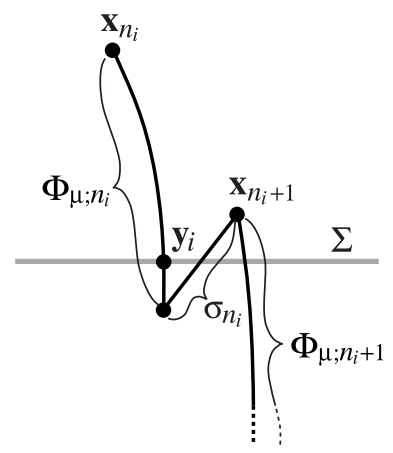

(a)

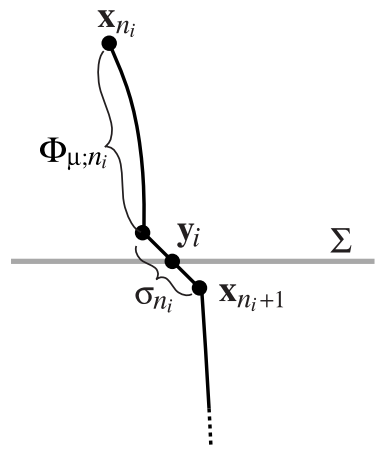

(b)

FiguRE 6 . In case (a), both $\Phi_{\mu ; n_{i}}, \Phi_{\mu ; n_{i}+1}$ meet $\Sigma$ non-trivially. But, the crossing point of $\Phi_{\mu ; n_{i}+1}$ with $\Sigma$ is not an element of $\left\{\mathbf{y}_{i}\right\}_{i \geq 0}$, i.e., $n_{i+1}>n_{i}+1$.

Remark 4.2. We note that a $(\delta, \hat{\tau})$-crossing sequence $\left\{\mathbf{y}_{i}\right\}_{i \geq 0}$ for $\varphi_{\mu}$ is in general not a pseudo-orbit for $L_{\mu}$ even if $\delta>0$ is very small. The crucial part in our proof of Theorem 2.7 is to show that $\left\{\mathbf{y}_{i}\right\}_{i \geq 0}$ is approximated by a pseudo-orbit $\left\{\mathbf{w}_{i}\right\}_{i \geq 0}$ for $L_{\mu}$, which in turn is approximated by an actual orbit $\left\{L^{i}(\mathbf{z})\right\}_{i \geq 0}$ of $L$ by Theorem 2.3 .

4.2. Proof of Theorem 2.7. For any $(\delta, \hat{\tau})$-crossing sequence $\left\{\mathbf{y}_{i}\right\}_{i \geq 0}$ for $\varphi_{\mu}$, the broken subsegment in $\bigcup_{n \geq 0} \tilde{\Phi}_{\mu ; n}$ connecting $\mathbf{y}_{i}$ with $\mathbf{y}_{i+1}$ is denoted by $\left\langle\mathbf{y}_{i}, \mathbf{y}_{i+1}\right\rangle_{\mu}^{\delta}$. In the case when $\left\{\mathbf{y}_{i}\right\}_{i \geq 0}$ is a finite sequence $\left\{\mathbf{y}_{i}\right\}_{i=0}^{m},\left\langle\mathbf{y}_{m},-\right\rangle_{\mu}^{\delta}$ is the broken forward ray in $\bigcup_{n \geq 0} \tilde{\Phi}_{\mu ; n}$ emanating from $\mathbf{y}_{m}$.

For any $\mathbf{z} \in \Sigma \backslash \Gamma$ and $\mu \in\left[0, \mu_{0}\right]$, let $\tau_{\mu ; \mathbf{z}}>0$ be the number with $\varphi_{\mu}\left(\mathbf{z},\left(0, \tau_{\mu ; \mathbf{z}}\right)\right) \cap$ $\Sigma=\emptyset$ and $\varphi_{\mu}\left(\mathbf{z}, \tau_{\mu ; \mathbf{z}}\right) \in \Sigma$, that is, $\varphi_{\mu}\left(\mathbf{z}, \tau_{\mu ; \mathbf{z}}\right)=L_{\mu}(\mathbf{z})$.

For any $0<\eta \leq 1$, set $\Pi(\eta)=[-\eta, \eta]^{2} \times[0, \eta], \partial_{\text {side }} \Pi(\eta)=\{-\eta, \eta\} \times[-\eta, \eta] \times[0, \eta]$ and $\partial_{\text {top }} \Pi(\eta)=[-\eta, \eta]^{2} \times\{\eta\}$. Note that $\partial_{\text {side }} \Pi(\eta)$ (resp. $\partial_{\text {top }} \Pi(\eta)$ ) consists of two vertical rectangles (resp. a single horizontal square) in $\Pi=\Pi(1)$; see Figure 7 Since any $(\delta, \hat{\tau})$-pseudo-orbit $\left\{\mathbf{x}_{n}\right\}_{n \geq 0}$ for $\varphi_{\mu}$ is taken in the trapping region $T_{\varphi_{\mu}}$ (see Definition 2.4), $\bigcup_{n \geq 0} \tilde{\Phi}_{\mu ; n} \cap \partial \Pi(\eta)$ is contained in $\partial_{\text {top }} \Pi(\eta) \cup \partial_{\text {side }} \Pi(\eta)$ for any small $\eta>0$, which is suggested by Figure 2 and Figure 7 . 


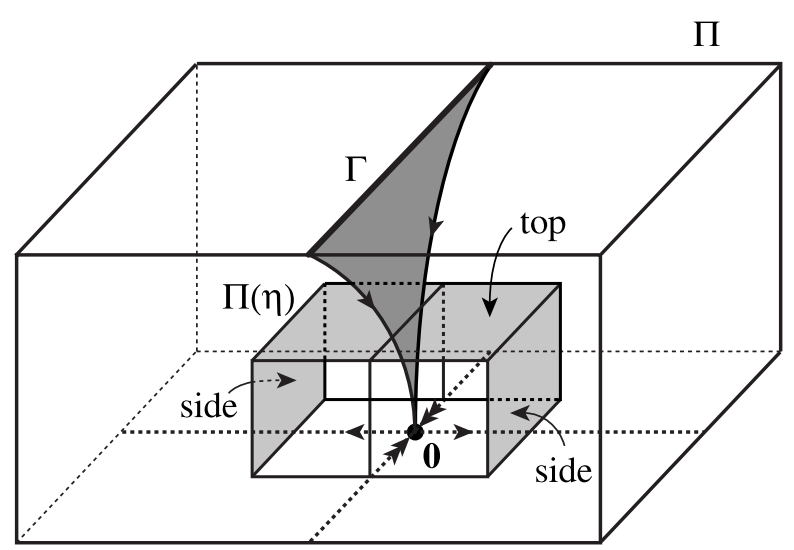

Figure 7. "side's" represent $\partial_{\text {side }} \Pi(\eta)$, and "top" represents $\partial_{\text {top }} \Pi(\eta)$. The gray cusp with vertex $\mathbf{0}$ is the union $\bigcup_{\mathbf{x} \in \Gamma} \varphi_{\mu}(\mathbf{x},[0, \infty)) \subset W^{s}(\mathbf{0})$.

Lemma 4.3. There exist $0<\delta_{2} \leq \delta_{0}, 0<\mu_{1} \leq \mu_{0}$ and $0<\varepsilon_{1}<1 / 10$ such that, for any $0<\mu<\mu_{1}$ and any $\left(\delta_{2}, \hat{\tau}\right)$-crossing sequence $\left\{\mathbf{y}_{i}\right\}_{i \geq 0}$ for $\varphi_{\mu}$, the following conditions (i) and (ii) hold:

(i) If $\mathbf{z}_{i} \in \Sigma \backslash \Gamma$ satisfies $\left|\mathbf{z}_{i}-\mathbf{y}_{i}\right| \leq \varepsilon_{1}$ and $\left|L\left(\mathbf{z}_{i}\right)-\mathbf{y}_{i+1}\right| \leq \varepsilon_{1}$, then $\left\langle\mathbf{y}_{i}, \mathbf{y}_{i+1}\right\rangle_{\mu}^{\delta_{2}}$ is $\varepsilon$-shadowed by $\varphi\left(\mathbf{z}_{i},\left[0, \tau_{0 ; \mathbf{z}_{i}}\right]\right)$.

(ii) If $\left\{\mathbf{y}_{i}\right\}_{i \geq 0}$ is a finite sequence $\left\{\mathbf{y}_{i}\right\}_{i=0}^{m}$, then for any $\mathbf{z}_{m} \in \Gamma$ with $\left|\mathbf{z}_{m}-\mathbf{y}_{m}\right| \leq$ $\varepsilon_{1},\left\langle\mathbf{y}_{m},-\right\rangle_{\mu}^{\delta_{2}}$ is $\varepsilon$-shadowed by $\varphi\left(\mathbf{z}_{m},[0, \infty)\right)$.

Proof. (i) From the definition of Lorenz flows, we have $0<\eta_{0} \leq \varepsilon / 30$ such that the restriction $\varphi_{\mu} \mid \Pi\left(3 \eta_{0}\right)$ is the linear flow $\left(e^{\lambda_{1} t} x, e^{-\lambda_{2} t} y, e^{-\lambda_{3} t} z\right)$ for some $-\lambda_{2}<$ $-\lambda_{3}<0<\lambda_{3}<\lambda_{1}$ independent of $\mu \in\left[0, \mu_{0}\right]$. There exists $\eta_{1}>0$ such that $\left|\left[\varphi_{\mu}(\mathbf{x},[0, \hat{\tau}])\right]_{x}\right|-\left|[\mathbf{x}]_{x}\right| \geq \eta_{1}$ for any $\mu \in\left[0, \mu_{0}\right]$ and any $\mathbf{x} \in \Pi$ with $\varphi_{\mu}(\mathbf{x},[0, \hat{\tau}]) \cap$ $\partial_{\text {side }} \Pi\left(\eta_{0}\right) \neq \emptyset$ and $[\mathbf{y}]_{y}-\left[\varphi_{\mu}(\mathbf{y},[0, \hat{\tau}])\right]_{y} \geq \eta_{1}$ for any $\mathbf{y} \in \Pi$ with $\varphi_{\mu}(\mathbf{y},[0, \hat{\tau}]) \cap$ $\partial_{\text {top }} \Pi\left(\eta_{0}\right) \neq \emptyset$; see Figure 8 Then, there exists $0<\delta_{1} \leq \min \left\{\eta_{0}, \eta_{1} / 2\right\}$ such that, for any interpolated $\left(\delta_{1}, \hat{\tau}\right)$-chain $\left\{\tilde{\Phi}_{\mu ; n}\right\}_{n \geq 0}$, if $\tilde{\Phi}_{\mu ; n} \cap \partial_{\text {side }} \Pi\left(\eta_{0}\right) \neq \emptyset$, then $\tilde{\Phi}_{\mu ; n+2} \cap \Pi\left(\eta_{0}\right)=\emptyset$. Intuitively, this means that the chain $\left\{\tilde{\Phi}_{\mu ; k}\right\}_{k>n}$ eventually goes away from $\Pi\left(\eta_{0}\right)$ if $\tilde{\Phi}_{\mu ; n} \cap \partial_{\text {side }} \Pi\left(\eta_{0}\right) \neq \emptyset$; see Figure 9. Similarly, one can choose the $\delta_{1}$ so that, if $\tilde{\Phi}_{\mu ; n} \cap \partial_{\text {top }} \Pi\left(\eta_{0}\right) \neq \emptyset$, then $\tilde{\Phi}_{\mu ; n+2} \cap \partial_{\text {top }} \Pi\left(\eta_{0}\right)=\emptyset$. For any $\mathbf{z} \in \Sigma \backslash \Gamma$, we set $t_{\mu ; \mathbf{z}}=0$ if $\varphi_{\mu}\left(\mathbf{z},\left[0, \tau_{\mu ; \mathbf{z}}\right]\right) \cap \Pi\left(\eta_{0}\right)=\emptyset$ and otherwise $t_{\mu ; \mathbf{z}}=\tau_{+}-\tau_{-}$, where $\left[\tau_{-}, \tau_{+}\right]$is the subinterval of $\left[0, \tau_{\mu ; \mathbf{z}}\right]$ with $\varphi_{\mu}\left(\mathbf{z},\left[\tau_{-}, \tau_{+}\right]\right)=\varphi_{\mu}\left(\mathbf{z},\left[0, \tau_{\mu ; \mathbf{z}}\right]\right) \cap \Pi\left(\eta_{0}\right)$.

Since $\varphi_{\mu}$ has no singular points in $T_{\mu} \backslash \Pi\left(\eta_{0}\right)$, there exists $s_{0}>0$ such that, for any $\mathbf{z} \in \Sigma \backslash \Gamma$ and $\mu \in\left[0, \mu_{0}\right], \tau_{\mu ; \mathbf{z}}-t_{\mu ; \mathbf{z}}<s_{0}$. From this, we know that, for any interpolated $\left(\delta_{1}, \hat{\tau}\right)$-chain $\left\{\Phi_{\mu ; n}\right\}_{n \geq 0}$, there exists the number of $n$ 's with $n_{i} \leq n \leq n_{i+1}$ such that $\tilde{\Phi}_{\mu ; n}$ is not wholly contained in $\Pi\left(\eta_{0}\right)$, and is bounded by a constant independent of $\mathbf{z} \in \Sigma \backslash \Gamma$ and $\mu \in\left[0, \mu_{0}\right]$. Then, one can choose $0<\delta_{2} \leq \delta_{1}, 0<\mu_{1} \leq \mu_{0}, 0<\varepsilon_{1}<1$ such that, for any $\left(\delta_{2}, \hat{\tau}\right)$-crossing sequence $\left\{\mathbf{y}_{i}\right\}_{i \geq 0}$ for $\varphi_{\mu},\left\langle\mathbf{y}_{i}, \mathbf{y}_{i+1}\right\rangle_{\mu}^{\delta_{2}} \backslash \Pi\left(\eta_{0}\right)$ is $\varepsilon$-shadowed by $\varphi\left(\mathbf{z}_{i},\left[0, \tau_{0 ; \mathbf{z}_{i}}\right] \backslash\left[v_{-}, v_{+}\right]\right)$if $0<\mu \leq \mu_{1},\left|\mathbf{z}_{i}-\mathbf{y}_{i}\right| \leq \varepsilon_{1}$ and $\left|L\left(\mathbf{z}_{i}\right)-\mathbf{y}_{i+1}\right| \leq \varepsilon_{1}$, where $\left[v_{-}, v_{+}\right]$is the subinterval (possibly empty) of $\left[0, \tau_{0 ; \mathbf{z}_{i}}\right]$ with $\varphi\left(\mathbf{z}_{i},\left[0, \tau_{0 ; \mathbf{z}_{i}}\right]\right) \cap \Pi\left(\eta_{0}\right)=\varphi\left(\mathbf{z}_{i},\left[v_{-}, v_{+}\right]\right)$. Since 


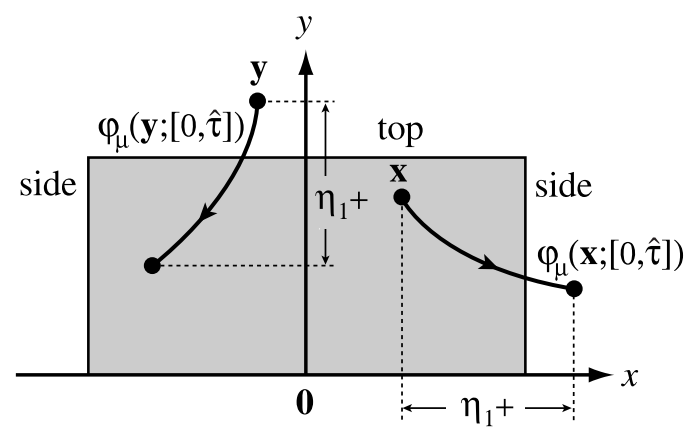

FiguRE 8 . The shaded rectangle represents $\Pi\left(\eta_{0}\right)$.

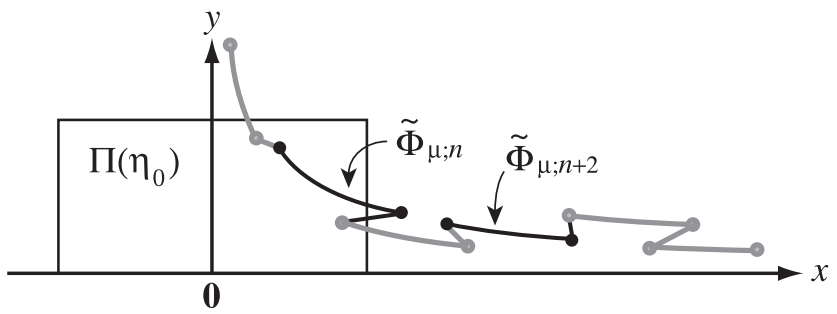

Figure 9

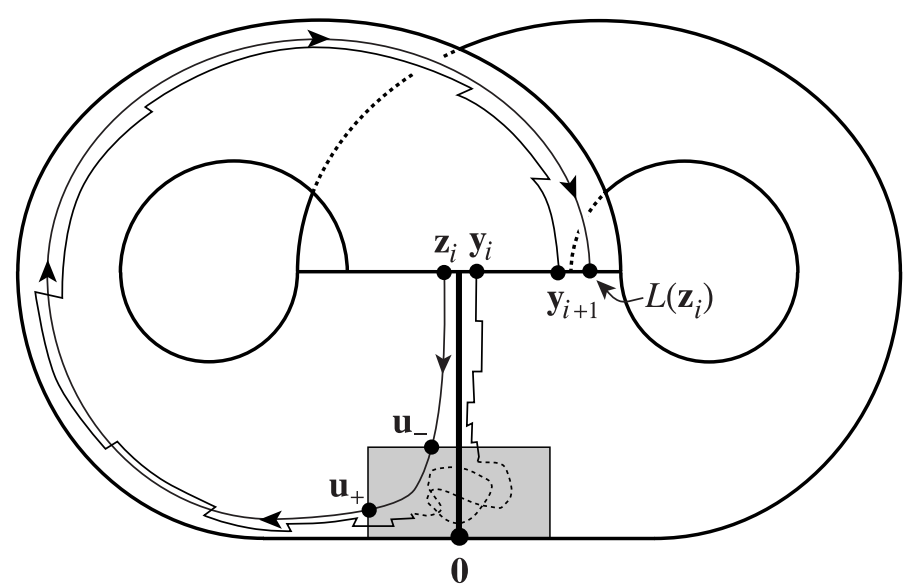

FiguRE 10. The points $\mathbf{u}_{ \pm}$represent $\varphi\left(\mathbf{z}_{i}, v_{ \pm}\right)$.

the diameter of $\Pi\left(\eta_{0}\right)$ is less than $\varepsilon / 2,\left\langle\mathbf{y}_{i}, \mathbf{y}_{i+1}\right\rangle_{\mu}^{\delta_{2}} \cap \Pi\left(\eta_{0}\right)$ is also $\varepsilon$-shadowed by $\varphi\left(\mathbf{z}_{i},\left[v_{-}, v_{+}\right]\right)$; see Figure 10. This shows the assertion (i).

(ii) The proof is quite similar to that of (i). Suppose that $0<\mu \leq \mu_{1}$ and $\left\{\mathbf{y}_{i}\right\}_{i=0}^{m}$ is a finite $\left(\delta_{2}, \hat{\tau}\right)$-crossing sequence for $\varphi_{\mu}$. From the argument in (i), $\left\langle\mathbf{y}_{m},-\right\rangle_{\mu}^{\delta_{2}}$ is disjoint from $\partial_{\text {side }} \Pi\left(\eta_{0}\right)$. For any $\mathbf{z}_{m} \in \Gamma$ with $\left|\mathbf{z}_{m}-\mathbf{y}_{m}\right| \leq \varepsilon_{1}$, let $v_{-}$be a unique point in $[0, \infty)$ with $\varphi\left(\mathbf{z}_{m}, v_{-}\right) \in \partial_{\text {top }} \Pi\left(\eta_{0}\right)$. Then, $\left\langle\mathbf{y}_{m},-\right\rangle_{\delta_{2}}^{\mu} \backslash \Pi\left(\eta_{0}\right)$ is $\varepsilon$-shadowed 
by $\varphi\left(\mathbf{z},\left[0, v_{-}\right]\right)$. Since $\varphi\left(\mathbf{z}_{i},\left[v_{-}, \infty\right)\right)$ is contained in $\Pi\left(\eta_{0}\right) \cap \tilde{\Gamma},\left\langle\mathbf{y}_{m},-\right\rangle_{\mu}^{\delta_{2}} \cap \Pi\left(\eta_{0}\right)$ is $\varepsilon$-shadowed by $\varphi\left(\mathbf{z}_{m},\left[v_{-}, \infty\right)\right)$. Thus, $\left\langle\mathbf{y}_{m},-\right\rangle_{\mu}^{\delta_{2}}$ is $\varepsilon$-shadowed by $\varphi\left(\mathbf{z}_{m},[0, \infty)\right)$.

By Theorem 2.3, there exist $\hat{\mu} \in\left(0, \mu_{1}\right]$ and $\xi_{0}>0$ such that any $\xi_{0}$-pseudo-orbit for $L_{\hat{\mu}}$ is $\varepsilon_{1} / 2$-shadowed by an actual orbit for $L$. From now on, we fix a $\hat{\mu}>0$ satisfying this condition and suppose that any pseudo-orbits and crossing sequences are those for $\varphi_{\hat{\mu}}$. Here, one can suppose that the $\xi_{0}$ is less than $\varepsilon_{1}$.

Proof of Theorem 2.7. First, let us consider the case when crossing sequences associated with a pseudo-orbits are infinite. We will show that there exists $0<\hat{\delta} \leq \delta_{2}$ such that, for the infinite crossing sequence $\left\{\mathbf{y}_{i}\right\}_{i \geq 0}$ associated with a $(\hat{\delta}, \hat{\tau})$-pseudoorbit $\left\{\mathbf{x}_{n}\right\}_{n \geq 0}$, there is an infinite sequence $\left\{\mathbf{w}_{i}\right\}_{i \geq 0}$ in $\Sigma$ which is a $\xi_{0}$-pseudo-orbit for $L_{\hat{\mu}}$ satisfying

$$
\left|\mathbf{y}_{i}-\mathbf{w}_{i}\right|<\varepsilon_{1} / 2
$$

for any $i \geq 0$.

Note that any flow of $\varphi_{\hat{\mu}}$ emanating from $\mathbf{0}$ tends toward either $\mathbf{v}_{+}$or $\mathbf{v}_{-}$. Take $0<\eta_{2} \leq \eta_{0}$ such that, for any $\mathbf{z} \in \Pi\left(\eta_{2}\right) \backslash \tilde{\Gamma}$, the first crossing point of $\varphi_{\hat{\mu}}(\mathbf{z}, t) ; t>0$ with $\Sigma$ is contained in either $N_{\xi_{0} / 3}\left(\mathbf{v}_{+}, \Sigma\right)$ or $N_{\xi_{0} / 3}\left(\mathbf{v}_{-}, \Sigma\right)$. There exists $0<\delta_{3} \leq \delta_{2}$ such that, for any $\left(\delta_{3}, \hat{\tau}\right)$-crossing sequence $\left\{\mathbf{y}_{i}\right\}_{i \geq 0}$, if $\left\langle\mathbf{y}_{i}, \mathbf{y}_{i+1}\right\rangle_{\hat{\mu}}^{\delta_{3}} \cap \Pi\left(\eta_{2}\right) \neq \emptyset$, then $\mathbf{y}_{i+1}$ is contained in either $N_{\xi_{0} / 2}\left(\mathbf{v}_{+}, \Sigma\right)$ or $N_{\xi_{0} / 2}\left(\mathbf{v}_{-}, \Sigma\right)$. Then, we have $0<\xi_{1} \leq \xi_{0} / 4$ and $0<\delta_{4} \leq \delta_{3}$ such that, for any $\left(\delta_{4}, \hat{\tau}\right)$-crossing sequence $\left\{\mathbf{y}_{i}\right\}_{i \geq 0}$, $\left\langle\mathbf{y}_{i}, \mathbf{y}_{i+1}\right\rangle_{\hat{\mu}}^{\delta_{4}}$ meets $\Pi\left(\eta_{2}\right)$ non-trivially if $\left|\left[\mathbf{y}_{i}\right]_{x}\right| \leq \xi_{1}$. One can take $0<\hat{\delta} \leq \delta_{4}$ such that if $\left|\left[\mathbf{y}_{i}\right]_{x}\right| \geq \xi_{1}$ for a $(\hat{\delta}, \hat{\tau})$-crossing sequence $\left\{\mathbf{y}_{i}\right\}_{i \geq 0}$, then $\left\langle\mathbf{y}_{i}, \mathbf{y}_{i+1}\right\rangle_{\hat{\mu}}^{\hat{\delta}}$ is disjoint from $\tilde{\Gamma}$ and

$$
\left|\mathbf{y}_{i+1}-L_{\hat{\mu}}\left(\mathbf{y}_{i}\right)\right|<\xi_{0} / 2 .
$$

We set $\mathbf{y}_{i}=\mathbf{w}_{i}$ if $\left|\left[\mathbf{y}_{i}\right]_{x}\right| \geq \xi_{1}$. Here, we need to consider the following three cases.

Case 1: $\left|\left[\mathbf{y}_{i}\right]_{x}\right| \geq \xi_{1}$ and $\left|\left[\mathbf{y}_{i+1}\right]_{x}\right| \geq \xi_{1}$.

Since $\mathbf{y}_{i}=\mathbf{w}_{i}$ and $\mathbf{y}_{i+1}=\mathbf{w}_{i+1}$, by (4.2), $\left|\mathbf{w}_{i+1}-L_{\hat{\mu}}\left(\mathbf{w}_{i}\right)\right|<\xi_{0}$.

Case 2: $\left|\left[\mathbf{y}_{i}\right]_{x}\right| \leq \xi_{1}$.

In this case, $\left\langle\mathbf{y}_{i}, \mathbf{y}_{i+1}\right\rangle_{\hat{\mu}}^{\hat{\delta}}$ may intersect with $\tilde{\Gamma}$ non-trivially. Then, it can happen that $\mathbf{y}_{i+1} \in N_{\xi_{0} / 2}\left(\mathbf{v}_{\iota}, \Sigma\right)$ and $L_{\hat{\mu}}\left(\mathbf{y}_{i}\right) \in N_{\xi_{0} / 2}\left(\mathbf{v}_{-\iota}, \Sigma\right)$ for some $\iota \in\{+,-\}$; see Figure 11 Take a point $\mathbf{w}_{i} \in \Sigma$ with $\left[\mathbf{w}_{i}\right]_{y}=\left[\mathbf{y}_{i}\right]_{y}$, $0<\left|\left[\mathbf{w}_{i}\right]_{x}\right| \leq \xi_{1}$ and $\iota=\operatorname{sign}\left[\mathbf{w}_{i}\right]_{x}=-\operatorname{sign}\left[\mathbf{y}_{i+1}\right]_{x}$. This definition implies $\left|\mathbf{w}_{i}-\mathbf{y}_{i}\right| \leq 2 \xi_{1} \leq \xi_{0} / 2<\varepsilon_{1} / 2$. Since $\left|\left[\mathbf{y}_{i+1}\right]_{x}\right| \geq 1-\xi_{0} / 2>\xi_{1}$, $\mathbf{y}_{i+1}=\mathbf{w}_{i+1}$ and hence

$$
\begin{aligned}
\left|L_{\hat{\mu}}\left(\mathbf{w}_{i}\right)-\mathbf{w}_{i+1}\right| & =\left|L_{\hat{\mu}}\left(\mathbf{w}_{i}\right)-\mathbf{y}_{i+1}\right| \\
& \leq\left|L_{\hat{\mu}}\left(\mathbf{w}_{i}\right)-\mathbf{v}_{\iota}\right|+\left|\mathbf{v}_{\iota}-\mathbf{y}_{i+1}\right| \\
& <\xi_{0} / 2+\xi_{0} / 2=\xi_{0}
\end{aligned}
$$

Case 3: $\left|\left[\mathbf{y}_{i}\right]_{x}\right| \geq \xi_{1}$ and $\left|\left[\mathbf{y}_{i+1}\right]_{x}\right|<\xi_{1}$.

As shown in the argument of Case 2, $\left|\mathbf{w}_{i+1}-\mathbf{y}_{i+1}\right| \leq 2 \xi_{1}$. Since $\mathbf{y}_{i}=\mathbf{w}_{i}$, the inequality (4.2) implies

$$
\begin{aligned}
\left|L_{\hat{\mu}}\left(\mathbf{w}_{i}\right)-\mathbf{w}_{i+1}\right| & \leq\left|L_{\hat{\mu}}\left(\mathbf{y}_{i}\right)-\mathbf{y}_{i+1}\right|+\left|\mathbf{y}_{i+1}-\mathbf{w}_{i+1}\right| \\
& <\xi_{0} / 2+2 \xi_{1} \leq \xi_{0} .
\end{aligned}
$$




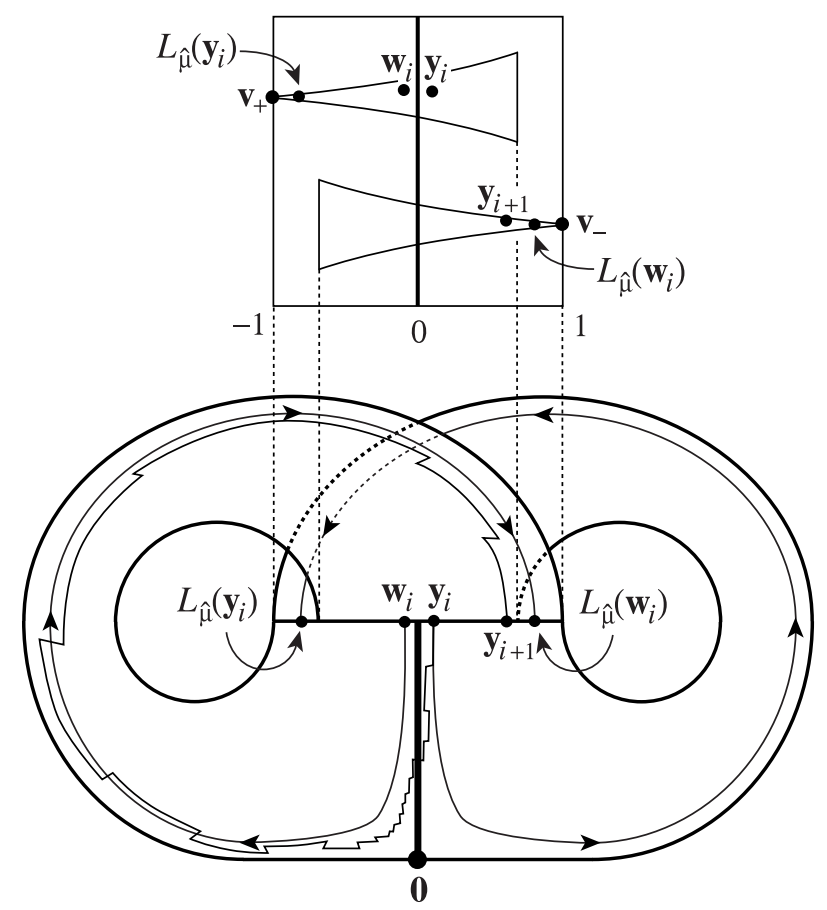

FIgURE 11. The case of $\left[\mathbf{y}_{i}\right]_{x}>0,\left[\mathbf{y}_{i+1}\right]_{x}>0$ and $\left[\mathbf{w}_{i}\right]_{x}<0$.

Then, $L_{\hat{\mu}}\left(\mathbf{y}_{i}\right)$ is not approximated by $\mathbf{y}_{i+1}$.

By Cases $1-3,\left\{\mathbf{w}_{i}\right\}_{i \geq 0}$ is a $\xi_{0}$-pseudo-orbit of $L_{\hat{\mu}}$ satisfying (4.1). By Theorem $2.3(\mathrm{i})$, there exists $\mathbf{z} \in \Sigma \backslash \Gamma$ with $\bigcup_{i=0}^{\infty} L^{i}(\mathbf{z}) \cap \Gamma=\emptyset$ and such that $\left\{L^{i}(\mathbf{z})\right\}_{i \geq 0}$ $\varepsilon_{1} / 2$-shadows $\left\{\mathbf{w}_{i}\right\}_{i \geq 0}$. Since $\left|\mathbf{y}_{i}-L^{i}(\mathbf{z})\right| \leq\left|\mathbf{y}_{i}-\mathbf{w}_{i}\right|+\left|\mathbf{w}_{i}-L^{i}(\mathbf{z})\right|<\varepsilon_{1}$, by Lemma 4.3(i), the $(\hat{\delta}, \hat{\tau})$-chain $\left\{\Phi_{\hat{\mu} ; n}\right\}_{n \geq 0}$ associated to $\left\{\mathbf{x}_{n}\right\}_{n \geq 0}$ is $\varepsilon$-shadowed by the actual orbit $\varphi(\mathbf{z}, t) ; t \geq 0$.

Next, we consider the case when crossing sequences associated with $\left(\delta_{2}, \hat{\tau}\right)$ pseudo-orbits is finite. By the argument as above, we have $0<\hat{\delta} \leq \delta_{2}$ such that, for the finite crossing sequence $\left\{\mathbf{y}_{i}\right\}_{i=0}^{m}$ associated with any $(\hat{\delta}, \hat{\tau})$-pseudoorbit $\left\{\mathbf{x}_{n}\right\}_{n \geq 0}$, there is a sequence $\left\{\mathbf{w}_{i}\right\}_{i=0}^{m}$ in $\Sigma$ which is a $\xi_{0}$-pseudo-orbit for $L_{\hat{\mu}}$ satisfying $\left|\mathbf{y}_{i}-\mathbf{w}_{i}\right| \leq \varepsilon_{1} / 2$ for any $i \in\{0,1, \cdots, m\}$. By Theorem 2.3 (ii), there exists $\mathbf{z} \in \Sigma$ with $\bigcup_{i=0}^{m-1} L^{i}(\mathbf{z}) \cap \Gamma=\emptyset, L^{m}(\mathbf{z}) \in \Gamma$ and such that $\left\{L^{i}(\mathbf{z})\right\}_{i=0}^{m} \varepsilon_{1} / 2$ shadows $\left\{\mathbf{w}_{i}\right\}_{i=0}^{m}$. Then, by applying Lemma 4.3(i) $(m-1)$-times and (ii) once, one can show that the $(\hat{\delta}, \hat{\tau})$-chain $\left\{\Phi_{\hat{\mu} ; n}\right\}_{n \geq 0}$ associated to $\left\{\mathbf{x}_{n}\right\}_{n \geq 0}$ is $\varepsilon$-shadowed by the actual orbit $\varphi(\mathbf{z}, t) ; t \geq 0$. This completes the proof of Theorem 2.7 .

\section{REFERENCES}

1. V.S. Afraimovich, V.V. Bykov, and L. P. Shil'nikov, On the appearance and structure of the Lorenz attractor, Dokl. Acad. Sci. USSR, 234 (1977) 336-339. MR 57:2150

2. D. V. Anosov, Geodesic flows on closed Riemann manifolds with negative curvature, Proc. Steklov Math. Inst., 90 (1967). MR 36:7157

3. W. J. Colmenarez and C. A. Morales, Transverse surfaces and attractors for 3-flows, Trans. Amer. Math. Soc., 354 (2002) 795-806. MR 2002h:37043 
4. C. M. Carballo, C. A. Morales and M. J. Pacifico, Maximal transitive sets with singularities for generic $C^{1}$ vector fields, Bol. Soc. Brasil. Mat. (N.S.), 31 (2000) 287-303. MR 2002b:37075

5. E. M. Coven, I. Kan and J. A. Yorke, Pseudo-orbit shadowing in the family of tent maps, Trans. Amer. Math. Soc., 308 (1988) 227-241. MR 90b:58236

6. J. Guckenheimer, A strange, strange attractor, in The Hopf bifurcation and its applications, (J. E. Marsden and M. McCracke eds.), Springer-Verlag, New York (1976). MR 58:13209

7. J. Guckenheimer and R.F. Williams, Structural stability of Lorenz attractors, Inst. Hautes Études Sci. Publ. Math., 50 (1979) 59-72. MR 82b:58055a

8. S. Kiriki and T. Soma, Parameter-shifted shadowing property of Lozi maps, preprint.

9. M. Komuro, Lorenz attractors do not have the pseudo-orbit tracing property, J. Math. Soc. Japan, 37 (1985) 489-514. MR 87a:58106

10. E. N. Lorenz, Deterministic non-periodic flow, J. Atmos. Sci., 20 (1963) 130-141.

11. C. A. Morales, Lorenz attractor through saddle-node bifurcations, Ann. Inst. H. Poincaré Anal. Non Linéaire, 13 (1996) 589-617. MR 97f:58084

12. C. A. Morales and M. J. Pacifico, Mixing attractors for 3-flows, Nonlinearity, 14 (2001) 359378. MR 2002a:37036

13. C. A. Morales, M.J. Pacifico and E.R. Pujals, Singular hyperbolic systems, Proc. Amer. Math. Soc., 127 (1999) 3393-3401. MR 2000c:37034

14. C. A. Morales, M. J. Pacifico and E. R. Pujals, Strange attractors across the boundary of hyperbolic systems, Comm. Math. Phys., 211 (2000) 527-558. MR 2001g:37036

15. H. E. Nusse and J. A. Yorke, Is every approximate trajectory of some process near an exact trajectory of a near process? Comm. Math. Phys., 114 (1988) 363-379. MR 89g:58108

16. S. Y. Pilyugin, Shadowing in dynamical systems, Lecture Notes in Math. 1706 SpringerVerlag (1999). MR 2001b:37030

17. W. Tucker, A rigorous ODE solver and Smale's 14th problem, Found. Comput. Math., 2 (2002) 53-117. MR 2003b:37055

18. M. Viana, What's new on Lorenz strange attractors? Math. Intelligencer, 22 (2000) 6-19. MR 2001h:37068

19. R. Williams, The structure of Lorenz attractors, Turbulence Seminar (Univ. Calif., Berkeley, Calif., 1976/1977), Lecture Notes in Math. 615 (pp. 94-112), Springer (1977). MR 57:1566

20. R. Williams, The structure of Lorenz attractors, Inst. Hautes Études Sci. Publ. Math., 50 (1979) 73-99. MR 82b:58055b

21. J. A. Yorke and E. D. Yorke, Metastable chaos: the transition to sustained chaotic behavior in the Lorenz model, J. Statist. Phys., 21 (1979) 263-277. MR 80f:76023

Department of Mathematical Sciences, Tokyo Denki University, Hatoyama, Hiki, SAITAMA-KEN, 350-0394, JAPAN

E-mail address: ged@r.dendai.ac.jp

Department of Mathematical Sciences, Tokyo Denki University, Hatoyama, Hiki, SAITAMA-KEN, 350-0394, JAPAN

E-mail address: soma@r.dendai.ac.jp 